\title{
Participant verification: Prevention of co-enrolment in clinical trials in South Africa
}

\author{
C Harichund, MSc; K Haripersad, MBA; G Ramjee, PhD \\ HIV Prevention Research Unit, Medical Research Council, Durban, South Africa
}

Corresponding author: C Harichund (charlene.harichund247@gmail.com)

\begin{abstract}
Background. As KwaZulu-Natal Province is the epicentre of the HIV epidemic both in South Africa (SA) and globally, it is an ideal location to conduct HIV prevention and therapeutic trials. Numerous prevention trials are currently being conducted here; the potential for participant co-enrolment may compromise the validity of these studies and is therefore of great concern.

Aim. To report the development and feasibility of a digital, fingerprint-based participant identification method to prevent co-enrolment at multiple clinical trial sites.

Methods. The Medical Research Council (MRC) HIV Prevention Research Unit (HPRU) developed the Biometric Co-enrolment Prevention System (BCEPS), which uses fingerprint-based biometric technology to identify participants. A trial website was used to determine the robustness and usability of the system. After successful testing, the BCEPS was piloted in July 2010 across 7 HPRU clinical research sites. The BCEPS was pre-loaded with study names and clinical trial sites, with new participant information loaded at first visit to a trial site. Results. We successfully implemented the BCEPS at the 7 HPRU sites. Using the BCEPS, we performed real-time 'flagging' of women who were already enrolled in another study as they entered a trial at an HPRU site and, where necessary, excluded them from participation on site Conclusion. This system has promise in reducing co-enrolment in clinical trials and represents a valuable tool for future implementation by all groups conducting trials. The MRC is currently co-ordinating this effort with clinical trial sites nationally.

S Afr Med J 2013;103(7):491-493. DOI:10.7196/SAMJ.6674
\end{abstract}

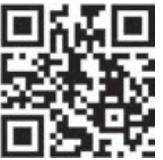

KwaZulu-Natal (KZN) Province has an estimated $39.5 \%$ HIV prevalence, ${ }^{[1]}$ making it both the national and global epicentre of the HIV epidemic. As a result, multiple clinical trials are conducted in the region. Co-enrolment is a risk, especially in poorly resourced communities, and has the potential to jeopardise participant safety and data integrity. Proposed reasons for co-enrolment include incentives, altruism and added synergistic benefit against HIV. ${ }^{[2]}$ Generally, study protocols that involve novel investigational products stipulate and enforce strict guidelines prohibiting co-enrolment. ${ }^{[3]}$

In 2008, 185 women were in violation of the exclusion criteria of the Centre for the AIDS Programme of Research in South Africa (CAPRISA) 004 and the HIV Prevention Trials Network (HPTN) 035 studies, both being conducted in KZN at research sites approximately $25 \mathrm{~km}$ apart. Women who co-enrolled in the 2 studies were subsequently exited from both studies prematurely. ${ }^{[2]}$ Reasons for co-enrolling, based on interviews, were largely attributed to the high-quality care and financial incentives received from trial participation. ${ }^{[4,5]}$ In response, to prevent further co-enrolment, the South African (SA) Medical Research Council (MRC) HIV Prevention Research Unit (HPRU) developed and implemented a co-enrolment system using a Microsoft Access database that could be utilised in the CAPRISA 004 and HPTN 035 studies.

The chief limitation of the Access database was that it did not provide real-time updates of participant information and was not linked to participant personal characteristics profiles. In addition, it was limited to a single point of verification for co-enrolment - the participant's identity document (ID) number - thus, the database was unable to recognise participants presenting false IDs or, in the case of identical twins, shared IDs. Lastly, the database was restricted to the MRC sites and 1 additional site.

We developed an electronic participant identification system, using biometric technology, to prevent co-enrolment and to ensure a unique and secure mechanism of identification that provided real-time updates. Here we report on the feasibility of this digital, fingerprintbased method to prevent co-enrolment at multiple clinical trial sites.

\section{Methods}

We determined the availability of digital identification systems through consultation with research organisations and external digital information technology (IT) consultants. The systems presented different points of verification: ID number-dependent only, fingerprint-dependent only and ID number plus fingerprint-dependent. Upon review, we concluded that none of these systems satisfied our requirements.

We conducted a 'needs assessment' at the 7 HPRU clinical research sites (CRSs) to identify criteria for the implementation of a biometric system. We then approached a software development company to develop a Biometric Co-enrolment Prevention System (BCEPS) according to the required specifications. A trial website was established; prospective BCEPS users were allowed access to test the system and to determine its robustness in handling different scenarios.

After successful testing, we gained approval from the relevant regulatory authorities and, with the assistance of the MRC IT Department, implemented the BCEPS at the 7 HPRU CRSs in July 2010. Information from the Access database was imported into the BCEPS. The BCEPS was password protected; only staff designated by the principal investigator (PI) were given access. Staff login was by fingerprint only, eliminating the sharing of usernames and passwords. Staff received extensive training on the use of the programme.

The BCEPS was preloaded with the names of studies and research sites. For fingerprint identification, the fingerprint in question had to be pre-loaded onto the database before a match could be returned. At first visit to a CRS, the participant's name, ID number and fingerprints were captured onto the BCEPS, the latter using a fingerprint reader. At subsequent visits, each participant's identity and study status was verified using their fingerprints. If the BCEPS showed that the participant was enrolled in another study, we concluded that co-enrolment was being attempted. 
Table 1. Comparison of the co-enrolment systems

\begin{tabular}{|c|c|c|}
\hline & MS Access database & BCEPS \\
\hline Location & Site and study specific & Web-based, linked across all CRSs \\
\hline Access to participant data & Site and study specific & $\begin{array}{l}\text { Linked across sites, all past and current } \\
\text { studies }\end{array}$ \\
\hline $\begin{array}{l}\text { Quality control of participant's information entered } \\
\text { at CRSs }\end{array}$ & Unable to generate quality-control reports & Daily quality-control reports generated \\
\hline Database access & Open to all staff & $\begin{array}{l}\text { Restricted fingerprint login access for } \\
\text { each staff member }\end{array}$ \\
\hline Point of verification for participants & ID number & ID number and fingerprints \\
\hline Back-up system ${ }^{*}$ & No back-up ${ }^{\dagger}$ & $3 \mathrm{G}$ card and laptop \\
\hline
\end{tabular}

The BCEPS was designed to allow access by multiple CRSs and research organisations in different provinces. A memorandum of understanding (MoU) and standard operating procedure is signed by all research organisations prior to BCEPS implementation.

\section{Results \\ BCEPS design and implementation}

After testing of the trial website, we determined that the infrastructure and running costs of implementation would include: a computer and fingerprint reader of approximately US\$2 000, an internet connection, and an annual fee of US $\$ 0.71$ per active record for system maintenance.

We implemented the BCEPS at one site at a time to ensure that the system functioned optimally. The fingerprint and profile of each user was required for login and needed to be entered into the system; this was timeconsuming, but only delayed implementation by a week. Staff experienced no challenges in learning how to use the programme. During the transition from the Access database to the BCEPS, some participants' fingerprints had to be captured for the new system to operate optimally; consequently, it took approximately 3 - 4 months for the old database to be phased out.

Challenges were encountered with the installation at some non-MRC sites: the BCEPS could not run on a 64-bit platform until an upgrade was performed; some organisations had to open hypertext transfer protocol secure (https) ports on their gateways before the BCEPS could be implemented and operate optimally. Where organisations had outsourced network administration, communication challenges between the outsourced company and MRC IT department required firewall reconfiguration. Additionally, the computers at some sites required upgrading to ensure

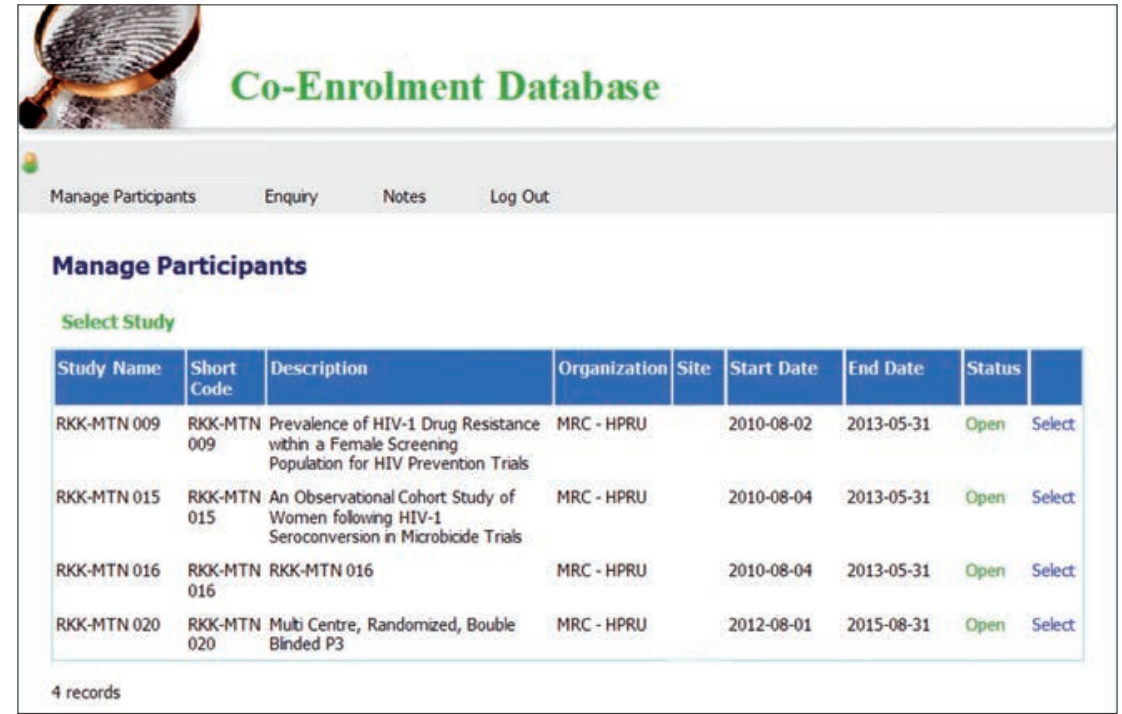

Fig. 1. Screenshot of the BCEPS demonstrating how the system was preloaded with the studies conducted at the 7 HPRU CRSs.

that the 'NET' framework was up to date and that the fingerprint reader was functional. Before signing the $\mathrm{MoU}$, external organisations were advised that a new computer should be purchased along with the fingerprint reader. When there was no internet connectivity, a back-up system comprising a laptop loaded with the BCEPS and a $3 \mathrm{G}$ card for internet access was available for the HPRU sites.

Difficulties in obtaining biometrics included obtaining fingerprints from participants with dry hands (necessitating moistening of the hands) and from smokers with indistinct fingerprints; this led to numerous attempts to capture at least 2 digits per hand.

To date, the system has approximately 21728 participants on the database with 129798 fingerprints recorded.

Real-time detection of co-enrolment Prior to BCEPS implementation, we preloaded all studies being conducted at a CRS on to the system (Fig. 1) and the study's PI had to approve access to all staff involved. All participants presenting at a CRS registered their details (name, surname, ID number and study name); these were entered into the participant registration log, creating a digital paper trail, and thereafter stored by the BCEPS. Fig. 2 illustrates the study participation history of a participant enrolled in a current HIV prevention clinical trial. The BCEPS detected that she was enrolled for 2 studies. In this case, the respective study protocols permitted the participant to be co-enrolled, since one study was an observational one and the other a prevention trial.

Participants who were found to be co-enrolled during the CAPRISA 004 and HPTN 035 clinical trials were 'flagged' as co-enrollers on the database by the MRC IT department at BCEPS implementation. When these women presented at the clinical 


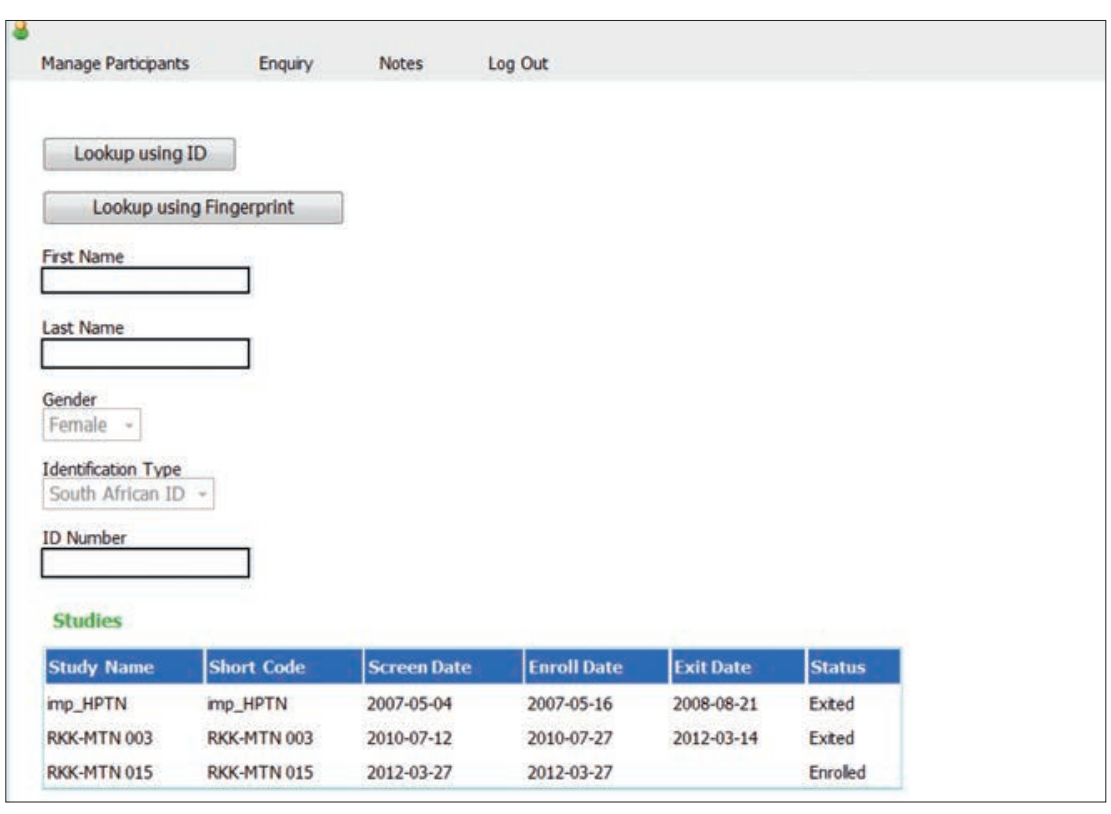

Fig. 2. Screenshot of the BCEPS demonstrating the study participation history of a participant enrolled in an HIV prevention clinical trial.

trial sites in 2010 for participation in a new clinical trial, the BCEPS detected their co-enrolment status and confirmed their ineligibility for participation.

The BCEPS is monitored and qualitycontrolled on a daily basis by comparing the CRS participant registration log with a BCEPS attendance report. The BCEPS proved comparatively more efficient than the Access database (Table 1) as it detected real-time co-enrolment; however, because the system is password protected to ensure participant confidentiality, thereby limiting access to designated staff only, staff were unable to edit any information once saved unless permitted by the 'super user'.

An external evaluation of the system performed by an independent company found that the system was accurate in its identification of participants at CRSs, was user-friendly, adaptable to any research environment and applicable to various computer operating systems.

\section{Discussion}

Most women who enrol in clinical trials are from impoverished settlements. Thus, the benefits they accrue from study participation - including free confidential relating to all clinic visits and verify real-time enrolment of a participant at an additional CRS.

We successfully tested and implemented the BCEPS at 7 HPRU sites and demonstrated that co-enrolment by participants at different clinical trial sites could be detected. Following set-up at the 7 HPRU CRSs, the BCEPS was established at the CAPRISA CRS. Having established the success of the system, we have subsequently been approached by other leading SA research organisations wishing to implement the BCEPS at their sites. The BCEPS is the first web-based technology to be used in SA with the aim of preventing co-enrolment in HIV prevention trials across multiple sites.

\section{Conclusion}

The biometric verification system is a novel approach to prevent participant co-enrolment in multiple HIV prevention clinical trials. Our study shows that the BCEPS is able to prevent such co-enrolment. The key to the system's success is the real-time updating of the participant database. The integrity of the system risks being compromised unless all research organisations, especially those in close proximity to each other, subscribe to a single biometric system such as the BCEPS. One major limitation is that the BCEPS is not presently linked to clinical trials that are being conducted by private organisations involving practitioners and pharmaceutical companies.

It is of paramount importance that organisations conducting clinical trials within the same regions implement a single system to prevent co-enrolment between research sites and studies. To our knowledge, there is limited global use of biometric technology in HIV prevention trials and elsewhere. In March 2012, the Department of Social Services launched a similar biometric fingerprinting system to curb fraud by people collecting their welfare grants. ${ }^{[6]}$

The clear advantages of biometric identification system are that it can: (i) provide extremely accurate, secured access to information; (ii) perform automated biometric identification rapidly and uniformly; (iii) verify a person's identity without resorting to documents that may be stolen, lost or altered; (iv) store information

\section{References}

1. Nel A, Mabude Z, Smit J, et al. HIV evidence remains high in KwaZulu-Natal, South Africa: Evidence from three districts. PLoS One 2012;7(4):e35278. [http://dx.doi.org/10.1371/journal. PLoS One 2012;

pone. 0035278

Abdool Karim QA, Kharsany AB, Naidoo K, et al. Co-enrolment in multiple HIV prevention trials - experiences from the CAPRISA 004 Tenofovir gel trial. Contemporary clinical trials 2011;32:333-338. [http://dx.doi.org/10.1016/j.cct.2011.01.005]

3. Nichol G, Powell JL, Emerson S. On co-enrolment in clinica resuscitation studies: Review and experience from randomized trials. Resuscitation 2010;81:792-795. [http://dx.doi.org/10.1016/j. resuscitation.2010.03.014]

4. Ramjee G, Coumi N, Dladla-Qwabe N, et al. Experiences in conducting multiple community-based HIV prevention trials among women in KwaZulu-Natal, South Africa. AIDS Research and Therapy 2010:7:10. [http///dx doi org/10.1186/1742-6405-7-10] and Therapy 2010,7:10. [1/P/dx.doi.or/10.1186/1742-6405-7-10 5. Naidoo K, Dorsamy E, Naidoo B, et al. Preventing co-enrolment in multiple clinical trials in Durban, South Africa. Abstract presented
at Microbicides, Cape Town, South Africa, 26 April 2006.

6. Timm S. New biometric card to boot out social grant fraud. Pretoria gcis.gov.za/news/12/12060813151001 (accessed 30 June 2012).

Accepted 8 April 2013. South African Government News Agency, 2012. http://oldsanews. 